\title{
THE DRIVE BEAM PULSE COMPRESSION SYSTEM FOR THE CLIC RF POWER SOURCE
}

\author{
R. Corsini, CERN, CH-1211 Geneva 23, Switzerland
}

\begin{abstract}
The Compact LInear Collider (CLIC) [1] is a high energy $(0.5$ to $5 \mathrm{TeV}) \mathrm{e}^{ \pm}$linear collider that uses a highcurrent electron beam (the drive beam) for $30 \mathrm{GHz} \mathrm{RF}$ power production by the Two-Beam Acceleration (TBA) method. Recently, a new cost-effective and efficient generation scheme for the drive beam has been developed [2,3]. A fully-loaded normal-conducting linac operating at lower frequency $(937 \mathrm{MHz})$ generates and accelerates the drive beam bunches, and a compression system composed of a delay-line and two combiner rings produces the proper drive beam time structure for RF power generation in the drive beam decelerator. In this paper, a preliminary design of the whole compression system is presented. In particular, the fundamental issue of preserving the bunch quality along the complex is studied and its impact on the beam parameters and on the various system components is assessed. A first design of the rings and delay-line lattice, including path length tuning chicanes, injection and extraction regions is also presented together with the simulation results of the beam longitudinal dynamics.
\end{abstract}

\section{DESCRIPTION OF THE SYSTEM}

A general layout of the bunch train compression system is shown in Figure 1. Its purpose is to split segments of the electron pulse delivered by the drive beam accelerator and later combine them in several stages in order to obtain 20 high-current pulses, each with 32 times the initial bunch repetition frequency and current. The system acts therefore both as a splitter and compressor as well as a frequency multiplier.

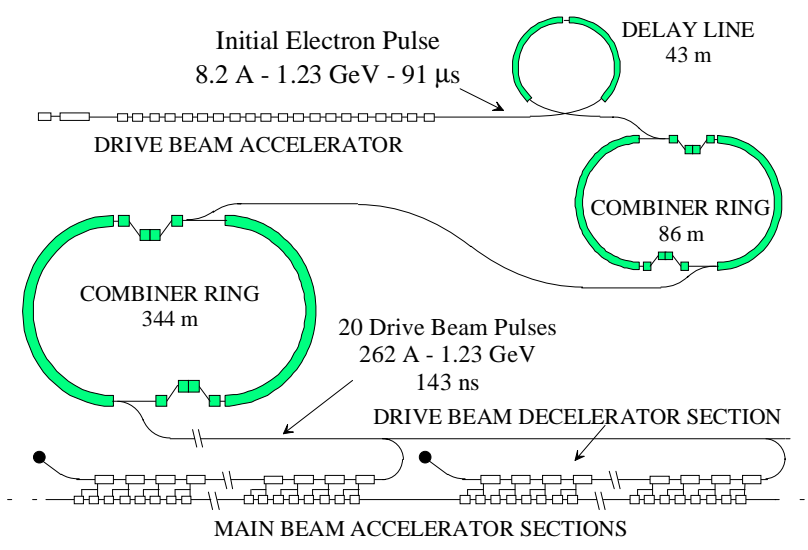

Figure 1: Layout of the drive beam compression system
The drive beam accelerator delivers $91 \mu$ s long electron pulses with a mean current of $8.2 \mathrm{~A}$ at $1.23 \mathrm{GeV}$. Each pulse is obtained by the combination at low energy of 640 bunch trains, $143 \mathrm{~ns}$ long, whose flat-top bunch charge is $17.6 \mathrm{nC}$. In each train the bunches occupy alternatively even and odd buckets of the drive beam accelerator fundamental frequency $(937 \mathrm{MHz})$. At first, the individual trains are separated by a transverse RF deflector at the frequency of $468.5 \mathrm{MHz}$, and each "even" bunch train is delayed with respect to the following "odd" one by $143 \mathrm{~ns}$, in order to recombine them using a second RF deflector at the same frequency. The whole device is called the "times-two delay combiner" (see Figure 1). After recombination, the pulse is composed of 320 trains whose spacing is equal to twice the train length ( $286 \mathrm{~ns}$, or $86 \mathrm{~m})$. The bunches fill all the buckets of the fundamental frequency $(320 \mathrm{~mm}$ spacing). The same principle of electron bunch train combination is then used to combine the trains four by four in the first combiner ring, $86 \mathrm{~m}$ long. Two $937 \mathrm{MHz}$ RF deflectors create a time-dependent local deformation of the equilibrium orbit in the ring. This bump is used for injection of a first train in the ring (all its bunches being deflected by the second RF deflector onto the equilibrium orbit). The ring length is equal to the spacing between trains plus $\lambda / 4$, where $\lambda$ is the spacing between bunches, equal to the wavelength of the RF deflectors. Thus for each revolution period the RF phase seen by the bunches circulating in the ring increases by $90^{\circ}$, and when the second train is injected, the first train does not see any deflection and its bunches are interleaved with the ones being injected (at a $\lambda / 4$ distance). This is repeated twice, then the four interleaved trains are extracted from the ring by an ejection kicker half a turn later, and the same cycle starts again. After the first combiner ring the whole pulse is composed of 80 trains. The train spacing is equal to eight times the train length (1144 ns or $344 \mathrm{~m}$ ), while the bunch spacing is $80 \mathrm{~mm}$.

The trains are combined again, using the same mechanism, in the second combiner ring, $344 \mathrm{~m}$ long, yielding another factor four in frequency multiplication, and obtaining the final 20 trains, with a bunch spacing of $20 \mathrm{~mm}$. The distance between trains is now $4.576 \mu$ s or $1375 \mathrm{~m}$, corresponding to twice the length of a drive beam decelerator section. This spacing is now correct to obtain proper phasing with the main beam pulse, distributing the drive beam trains along the decelerator in the opposite direction to the main beam. 


\section{DESIGN ISSUES}

The main problem to be solved in the system is to preserve the bunch quality during the compression. In particular, bunch length and longitudinal phase-space distribution must be preserved and train to train, bunch to bunch fluctuations in phase and transverse position minimised. The rings and the delay line must then be isochronous.

The final bunch length must be short in order to maximise the $30 \mathrm{GHz}$ RF power production efficiency in the drive beam decelerator. At present the aim is a rms bunch length of $\sigma=0.4 \mathrm{~mm}$ (94\% efficiency). Such high-charge $(17.6 \mathrm{nC})$, short bunches can radiate coherently a considerable amount of synchrotron radiation, leading both to an average energy loss of the whole bunch and to an energy spread within the bunch itself [4]. The emission is concentrated at low frequencies $(v \leq 1 /(2 \sigma))$, and can be partly suppressed if at least part of the emitted spectrum is below cutoff in the beam pipe (shielding effect), but a limit is imposed by the necessary beam clearance. Since bunches belonging to different trains make a different number of turns in the rings (from $1 / 2$ to $7 / 2$ ), they will develop different energy distributions. This will cause relative phase errors between bunches, bunch lengthening and phase-space distortion through non-perfect isochronicity of the delay line and the combiner rings. Short, highcharge bunches will also interact with any small discontinuity of the beam chamber (e.g., bellows and septa), possibly being subject to longitudinal and transverse wake-fields. It is therefore highly desirable to manipulate relatively long bunches in the compression system and compress them just before injection into the drive beam decelerator sections. However, too long bunches experience a non-uniform kick in the RF deflectors, due to their phase extension, and the singlebunch emittance can grow. Therefore the bunch length at the exit of the accelerator is limited to $2 \mathrm{~mm}$ rms. The maximum emittance growth in this case is $\sim 2 \%$ (for an initial rms normalized emittance of $2 \times 10^{-4} \pi \mathrm{m} \mathrm{rad}$ ), in the second combiner ring deflectors $(3.75 \mathrm{GHz})$. A correlated energy spread $(\sim 1 \% \mathrm{rms})$, suitable for the final bunch compression, is obtained in the accelerator by the combined effect of RF curvature and longitudinal wake-fields. The need to preserve the correlation all along the compression system means that all the distortions of the longitudinal phase space must be kept small. In particular, attention must be given to the higher orders of the path length dependence on energy spread. A numerical analysis has shown that second order effects will hamper the final bunch compression and must be corrected by using sextupoles [3].

Another concern is the beam loading in the RF deflectors, especially just before extraction in the second combiner ring, where the average current along the train is very high and the frequency of the deflectors
(3.75 GHz) is the highest in the compression system. In order to overcome this problem, a travelling-wave irisloaded structures with a short filling time with respect to the train duration has been chosen. A steady state condition is then reached with minimum transient effects, although at the expenses of a loss in deflection efficiency. The extraction system for both rings is also a critical item, the issues being the high repetition rate (particularly in the first ring - $250 \mathrm{kHz}$ ) and the interaction with the high-current beam (particularly in the second ring $-262 \mathrm{~A}$ ). The solution adopted for both rings is based on the use of travelling TEM wave transmission line pairs [5].

\section{SUB-SYSTEMS AND COMPONENTS}

In Table 1 and Table 2 the parameters of the sub-systems are presented. The lengths of the rings are fixed by the overall timing; the delay combiner geometry has been chosen to minimise both coherent synchrotron radiation emission and overall dimensions. One 3-bend magnetic chicane is located in the delay line and one in each ring arc. They are used for path length tuning $( \pm 0.5 \mathrm{~mm}$ tuning range), in order to adjust the bunch relative phase and to compensate for orbit variations. Each chicane is $3.5 \mathrm{~m}$ long and works around an average bending angle $\theta_{0} \sim 150 \mathrm{mrad}$. The tuning range is obtained with a bending angle variation $\Delta \theta=1.5 \mathrm{mrad}$; such a small value of $\Delta \theta / \theta_{0}$ does not perturb the optics. Each chicane has a linear transfer matrix element $R_{56}=0.065$.

Table 1: Delay combiner parameters

\begin{tabular}{|l|c|c|}
\hline Length (line 1 / 2) & $25 / 68$ & $\mathrm{~m}$ \\
\hline Bending radius (line 2) & 3 & $\mathrm{~m}$ \\
\hline \# Dipoles (line 2) & 16 & \\
\hline Dipole length & 2 & $\mathrm{~m}$ \\
\hline Dipole Field & 1.25 & $\mathrm{~T}$ \\
\hline
\end{tabular}

Table 2: Rings parameters

\begin{tabular}{|l|c|c|c|}
\hline & Ring 1 & Ring 2 & \\
\hline Circumference & 86 & 344 & $\mathrm{~m}$ \\
\hline Bending radius & 3.6 & 17.8 & $\mathrm{~m}$ \\
\hline \# Cells & 4 & 20 & \\
\hline Cell length & 13.6 & 13.6 & $\mathrm{~m}$ \\
\hline \# Dipoles & 16 & 80 & \\
\hline Dipole length & 1.4 & 1.4 & $\mathrm{~m}$ \\
\hline Dipole field & 1.1 & 0.22 & $\mathrm{~T}$ \\
\hline \# Quadrupoles & 52 & 184 & \\
\hline Quadrupole length & 0.3 & 0.3 & $\mathrm{~m}$ \\
\hline Max quad gradient & 14 & 14 & $\mathrm{~T} / \mathrm{m}$ \\
\hline \# Sextupoles & 20 & 84 & \\
\hline Sextupole length & 0.3 & 0.3 & $\mathrm{~m}$ \\
\hline Max sext gradient & 26 & 120 & $\mathrm{~T} / \mathrm{m}^{2}$ \\
\hline Max $\beta$-function (h,v) & $10.3,10.9$ & $32.6,16.8$ & $\mathrm{~m}$ \\
\hline
\end{tabular}




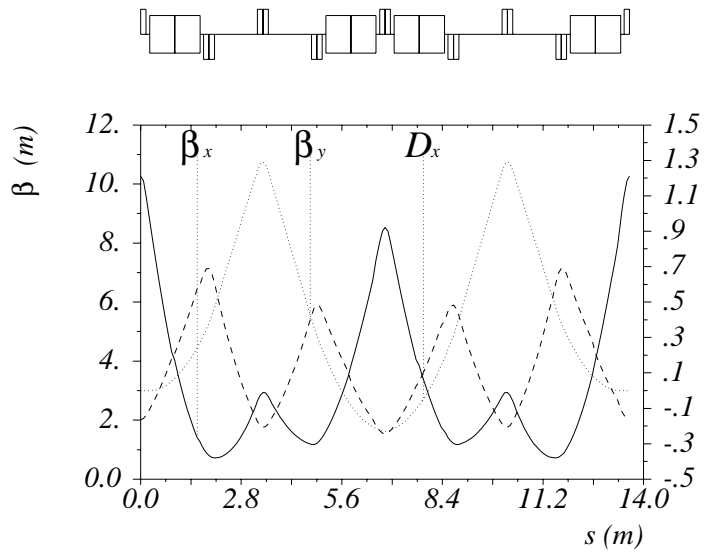

Figure 2: Basic isochronous cell lattice

Both the delay line and the ring arcs are based on the same type of isochronous lattice cell (see Figure 2), a modified four-cell FODO structure with "missing magnets" [6]. If other elements in the lattice (i.e., the chicanes) have a small nonzero $R_{56}$, the cell can be tuned to be quasi-isochronous, its residual $R_{56}$ being such that the sum of the individual contributions is zero.

In order to avoid distortions in the longitudinal phase space, all the arcs are made isochronous up to second order by the use of sextupoles placed in the highdispersion regions in correspondence with the missing magnets. By using different families of sextupoles it is possible to correct the third order as well. In principle, it should also be possible to tune the path length dependence from the energy spread (the isochronicity curve), in such a way as to compensate distortions in the longitudinal phase space of the electron bunches due to other non-linear effects. The ring injection is similar to a conventional fast injection scheme based on a septum and a fast kicker, where the kicker's role is taken by a $\mathrm{RF}$ deflector. Another deflector is placed upstream of the septum (at $-\pi$ phase advance), and provides the precompensation of the kick given by the injection deflector to the circulating bunches. A $\pi / 2$ phase advance FODO lattice is used in the injection straight section, with the septum and deflectors close to the focusing quadrupoles, such that the angular kick from the deflector corresponds to a maximum displacement in the septum. All RF deflectors are short travelling wave iris-loaded structures, whose fundamental mode is a deflecting hybrid mode with a $2 \pi / 3$ phase advance per cell and negative group velocity [7]. The design is basically the same for all deflectors, with the dimensions of the cells scaled linearly with frequency. They are made up of 4 cells at $937 \mathrm{MHz}$ and of 10 cells at $3.75 \mathrm{GHz}$ and provide the needed 2 mrad deflection, equal in both rings, with $50 \mathrm{MW}$ and $15 \mathrm{MW}$ power consumption, respectively. The extraction kickers consist of travelling TEM wave transmission line pairs [5], powered in antiphase, with the wave moving against the beam; the kicker length is chosen to be $2 \mathrm{~m}$ with a half-aperture of
$1.5 \mathrm{~cm}$. A deflection of $3 \mathrm{mrad}$ requires a voltage of 11.3 $\mathrm{kV}$, corresponding to $2.6 \mathrm{MW}$ into each $50 \Omega$ line. The kicker filling time of $6 \mathrm{~ns}$ remains small compared with the $143 \mathrm{~ns}$ available rise time. Due to the length of the extraction kicker, a different solution is used for the extraction region lattice, based on matching quadrupole doublets plus a triplet placed between the kicker and the extraction septum. The phase advance between the kicker and the septum is still $\sim \pi / 2$, and the use of a central triplet allows a rather constant $\beta$-function along the kicker to be obtained.

\section{BEAM DYNAMICS}

As mentioned earlier, the preservation of the longitudinal phase-space of the bunches is important in order to be able to compress them before injection in the drive beam decelerator. The main sources of phase-space distortion in the compressor system chain are the coherent synchrotron radiation (CSR) emission and the higher-order non-isochronicity in the arcs. The bunch longitudinal dynamics in the both rings has been evaluated taking into account the CSR effect with shielding and the isochronicity curves for the arcs after sextupole correction up to second order [3]. The results are promising; the final bunch length after compression is smaller than the target one, with small bunch to bunch variation in final length (from 340 to $360 \mu \mathrm{m} \mathrm{rms}$, depending on the number of turns in the rings). The contribution of the delay combiner arcs, as well as the non-linear contributions from the return arcs and the final compressor, have been neglected for the moment. Nevertheless, these contributions are smaller, and further improvement can be obtained, e.g., either by using different sextupole families in order to compensate nonlinearities, or by adding pulse stretchers and compressors in front of each ring and optimizing the bunch length in each component.

\section{REFERENCES}

[1] J.P.Delahaye and 30 co-authors, "CLIC, a 0.5 to $5 \mathrm{TeV}$ e \pm Compact Linear Collider", Proc. EPAC'98, June 22-26 1998, Stockholm, Sweden, CERN/PS 98-09, and CLIC Note 360 (1998).

[2] H.H. Braun and 14 co-authors, "A New Method of RF Power Generation for Two-Beam Linear Colliders", Proc. EPAC'98, June 22-26 1998, Stockholm, Sweden, CERN/PS/98-14 (LP) and CLIC Note 367 (1999).

[3] H.H. Braun and 14 co-authors, "The CLIC RF Power Source, a Novel Scheme of Two Beam Acceleration for e \pm Linear Colliders", to be published as a CERN yellow report.

[4] J.B. Murphy, S. Krinsky and R.L. Gluckstern, "Longitudinal Wakefield for an Electron Moving on a Circular Orbit", Part. Acc. 57 (1997) 9.

[5] L. Thorndahl, CERN, Private Communication.

[6] R.C. York and D.R. Douglas, "Optics of the CEBAF CW Superconducting Accelerator", Proceedings of the 12th Particle Accelerator Conference, March 6-19, 1987, Washington, DC.

[7] $\mathrm{Ph}$. Bernard, H. Lengeler and V. Vaghin, "New Disk-Loaded Waveguides for the CERN RF Separator", CERN 70-26 (1970). 